\title{
Unusual intracranial suppuration: illustrative cases
}

\author{
Anjali Pradhan, BA, ${ }^{1,2}$ François Xavier Rutayisire, MD, ${ }^{3}$ Paulin Munyemana, MD, ${ }^{3}$ and Claire Karekezi, $\mathrm{MD}^{3}$ \\ ${ }^{1}$ Department of Neurosurgery, Ronald Reagan UCLA Medical Center, University of California, Los Angeles, California; ${ }^{2}$ David Geffen School of Medicine, Ronald Reagan UCLA \\ Medical Center, University of California, Los Angeles, Los Angeles, California; and ${ }^{3}$ Neurosurgery Unit, Department of Surgery, Rwanda Military Hospital, Kigali, Rwanda
}

\begin{abstract}
BACKGROUND Intracranial suppuration (ICS) is a rare complication that can arise from various disease processes and is composed of brain abscess, extradural empyema, and subdural empyema. Although significant progress has been achieved with antibiotics, neuroimaging, and neurosurgical technique, ICS remains a serious neurosurgical emergency. An uncommon presentation of ICS is sterile ICS, which has yet to be fully elucidated by clinicians. The authors present 2 cases of unusual sterile ICS: a sterile subdural empyema and a sterile brain abscess.

OBSERVATIONS Both patients underwent surgical treatment consisting of craniotomy to evacuate the pus collection. The blood cultures from both the patients, the collected empyema, and the thick capsule from the brain abscess were sterile. However, the necrotic brain tissue surrounding the abscess contained inflammatory cells. The authors' review of the literature emphasizes the rarity of sterile ICS and substantiates the necessity for additional studies to explore this field.
\end{abstract}

LESSONS Sterile ICS is a disease entity that warrants further investigation to determine appropriate treatment to improve patient outcomes. This study highlights the paucity of data available regarding sterile ICS and supports the need for future studies to uncover the etiology of sterile ICS to better guide management of this condition.

https://thejns.org/doi/abs/10.3171/CASE21570

KEYWORDS intracranial suppuration; sterile intracranial suppuration; subdural empyema; brain abscess

Intracranial suppuration (ICS) is a rare, life-threatening condition that consists of brain abscess (BA), extradural empyema, and subdural empyema (SDE). ${ }^{1-7}$ Prior to the inception of antibiotics, ICS was associated with nearly $100 \%$ morbidity and mortality in patients who presented for treatment within $24-48$ hours. ${ }^{6,8}$ Despite advances in modern neurosurgical technique, diagnostic neuroimaging, and new antibiotic therapies, ICS is still considered to be a dangerous neurosurgical emergency due to its potentially lethal nature. However, with prompt diagnosis and appropriate management, ICS is a treatable condition, and mortality rates have dropped to 4-9\%. ${ }^{9,10}$ In low- and middle-income countries, early diagnosis and treatment can be challenging due to limited access to medical facilities. In Africa, it is reported that $2-5 \%$ of all intracranial space-occupying lesions are composed of focal ICSs. ${ }^{4,11}$ Outside of Africa, in Western and developed countries, the incidence of focal ICSs has decreased to $0.4-0.6$ per $100,000 .^{12}$ Although improvements in neurosurgical technique and antimicrobial therapy have contributed to this significant decline in ICS incidence, the availability of and access to modern diagnostic neuroimaging techniques such as computed tomography (CT) are also largely associated with enhanced clinical outcomes. $^{1,4,13-15}$ The etiology of ICS typically results as a complication of numerous disease processes, including paranasal sinusitis, mastoiditis, osteomyelitis of the skull, head trauma with open fracture of the skull, prior cranial surgery, infected hematoma or subdural effusion, otitis, meningitis, bacterial endocarditis, or metastatic spread from distant foci. ${ }^{2,4,16-19}$ However, in some cases, the source of ICS cannot be determined. CT of the brain is considered the main imaging modality for detection and diagnosis of ICS; in cases where an accurate diagnosis cannot be achieved, magnetic resonance imaging (MRI) is used. $2,7,17,20-22$

Although SDE and BA are well-known disease entities of ICS, sterile site ICS is uncommonly seen. Therefore, the authors of this

ABBREVIATIONS BA = brain abscess; $\mathrm{CRP}=\mathrm{C}$-reactive protein; $\mathrm{CT}=$ computed tomography; $\mathrm{GCS}=$ Glasgow Coma Scale; HIV = human immunodeficiency virus; $I C P=$ intracranial pressure; $I C S=$ intracranial suppuration; MRI = magnetic resonance imaging; SDE = subdural empyema.

INCLUDE WHEN CITING Published December 13, 2021; DOI: 10.3171/CASE21570.

SUBMITTED October 5, 2021. ACCEPTED October 22, 2021.

(c) 2021 The authors, CC BY-NC-ND 4.0 (http://creativecommons.org/licenses/by-nc-nd/4.0/). 
study report 2 cases of sterile ICS, a sterile SDE and a aseptic occipital BA that were treated in the Neurosurgery Unit at Rwanda Military Hospital.

\section{Illustrative Cases}

Case 1

Preoperative History and Examination

A 33-year-old male presented with symptoms of right-sided body weakness and confusion that had begun a few weeks prior to seeking medical treatment. The patient had no significant past medical history, history of trauma, or sinusitis and was otherwise healthy. The results of hepatitis $\mathrm{C}$, hepatitis $\mathrm{B}$, and human immunodeficiency virus (HIV) tests were all negative. Of note, the patient was a prisoner. On examination, the patient was noted to have tattoos present bilaterally on his upper extremities; he was afebrile with a temperature of $36^{\circ} \mathrm{C}$; and the remainder of his vital signs on admission were unremarkable. Neurological examination revealed right-sided hemiplegia and a Glasgow Coma Scale (GCS) score of 13. His blood laboratory test results, including neutrophil count, were within normal limits, and his initial C-reactive protein (CRP) was $5 \mathrm{mg} / \mathrm{L}$. His brain CT scan revealed a left-sided subdural hypodense collection with significant mass effect on the ipsilateral ventricle and midline shift; the collection showed peripheral enhancement on contrast-enhanced CT (Fig. 1).

\section{Intraoperative Course}

The patient was subsequently admitted on the same day, and surgical treatment consisted of aspiration via 2 burr holes, which revealed the collection to be thick pus. A small minicraniotomy was then performed, and the pus was evacuated, as seen in Fig. 1. The finding of pus analysis was that the pus was sterile, and the result of GeneXpert analysis was negative for Mycobacterium tuberculosis.

\section{Postoperative Course and Follow-Up}

The patient was empirically treated with antimicrobial therapy consisting of ceftriaxone, cloxacillin, and metronidazole. In addition, he received phenytoin for seizure prevention. During the course of the patient's stay at our institution, he remained stable. After the surgical procedure, the patient improved significantly; he was ambulating, he had a GCS score of 15 , and his hemiplegia resolved. The patient remained at our institution for 1 month until he was discharged with oral antibiotic therapy consisting of amoxicillin and clavulanate (Augmentin; GlaxoSmithKline) $625 \mathrm{mg}$ 8-hourly for 2 weeks and remained on antiseizure prophylaxis. Therefore, the patient received a total of 6 weeks of antibiotic treatment. He was seen for follow-up 1 month after discharge in our outpatient clinic, and he had a complete neurological recovery. His postoperative brain CT scan showed good resolution and evacuation of the empyema (Fig. 2). His last CRP test result at 1 -month follow-up was $2 \mathrm{mg} / \mathrm{L}$.

\section{Case 2}

Preoperative History and Examination

A 42-year-old male with a past medical history significant for tuberculosis presented with 8 weeks of worsening symptoms of chronic headache, vomiting, blurry vision, and dizziness. To manage his headache, the patient was taking paracetamol at home; however, when new-onset symptoms of blurry vision and dizziness began, the patient presented to our institution for medical treatment and investigation. Upon consultation and neurological examination,
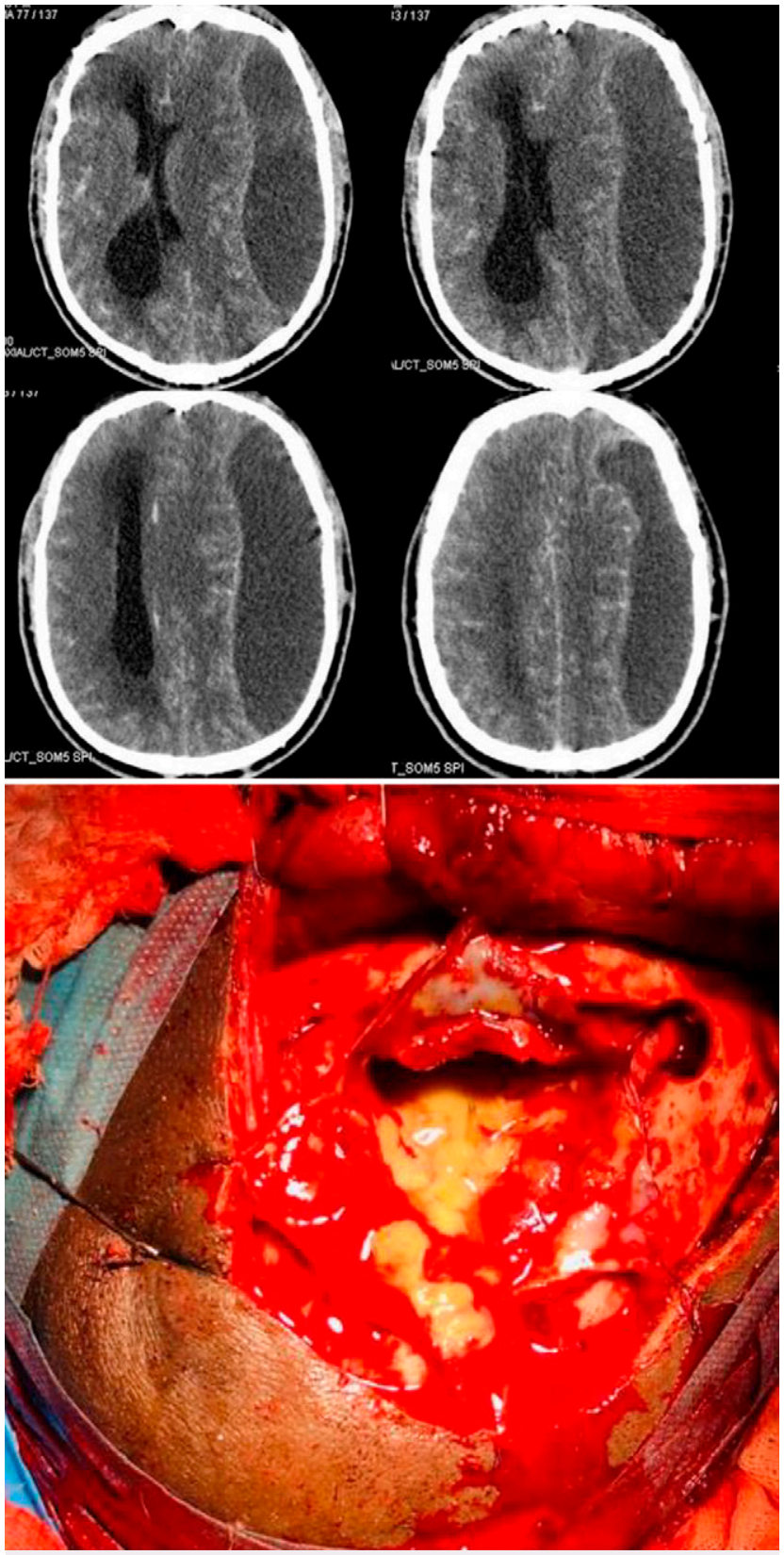

FIG. 1. Upper: Preoperative axial cut CT of the brain with intravenous (IV) contrast shows a left-sided subdural hypodense collection with significant mass effect on the ipsilateral ventricle, and midline shift was observed. The collection showed peripheral enhancement after IV contrast administration. Lower: Intraoperative aspiration of the hypodense collection was performed via craniotomy. Intraoperative findings revealed the collection to be thick pus with a thickening of the dura mater.

the patient was found to have right homonymous hemianopia suggestive of a probable space-occupying lesion with increased intracranial pressure (ICP). The patient was afebrile with a temperature of $36^{\circ} \mathrm{C}$ and had a blood pressure of $120 / 60 \mathrm{~mm} \mathrm{Hg}$, and the remainder of his vital signs on admission were within normal limits. On fundoscopic examination, the patient was found to have bilateral 


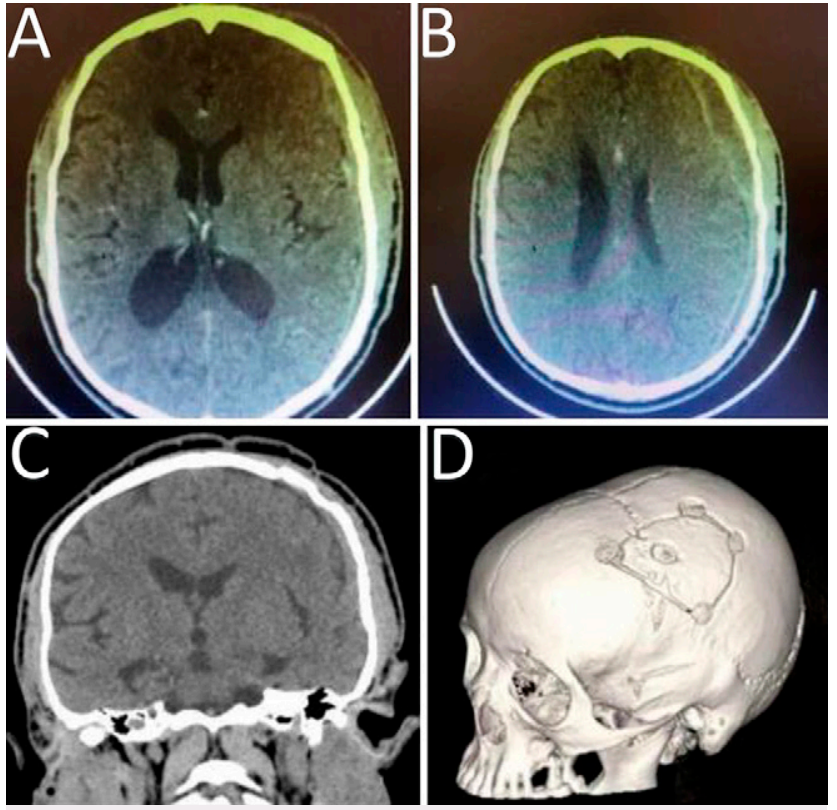

FIG. 2. A and B: Postoperative/follow-up axial cut brain CT scans show good evacuation and decompression of the subdural empyema after craniotomy. The small left-sided residual empyema was further treated with antibiotic therapy. C: Postoperative coronal cut brain CT scan. D: Bone window view of brain CT scan shows the position of the left-sided craniotomy procedure.

papilledema. The patient had a medical history of tuberculosis 7 years prior and was subsequently treated and declared healed. No other notable medical issues, including cardiac diseases, hepatitis, HIV, sinusitis, or history of trauma, were documented. Hepatitis C, hepatitis $B$, and HIV test results were all negative. On examination of his visual acuity, the patient had 20/40 vision in his right eye and $20 / 50$ vision in his left eye. His neck was soft and supple with no signs of nuchal rigidity. An electrocardiogram and cardiac ultrasound were performed and showed no evidence of vegetations or endocarditis. The patient was found to have a left-sided occipital abscess as seen on a preoperative MRI brain scan (Fig. 3).

\section{Intraoperative Course}

The surgical operation was performed in a right lateral position. A left occipital craniotomy was made, the dura was opened, and a thick capsule containing frank pus was found during the procedure (Fig. 4). More than $100 \mathrm{ml}$ of pus was drained from the patient's abscess. The capsule was carefully dissected and removed, the area was irrigated and washed, and hemostasis was achieved with Surgicel (Fig. 4).

\section{Postoperative Course and Follow-Up}

The capsule sent for pathology measured $45 \times 40 \mathrm{~mm}$ and was described to be a soft, tan-brownish tissue fragment. The capsule was surrounded by necrotic brain tissue, which the pathology report noted had findings of reactive astrocytes and inflammatory cells, mainly neutrophils, polymorphs, lymphocytes, and macrophages. However, no neoplastic processes were seen. Interestingly, the laboratory test results obtained from the capsule showed only 1 white blood cell. The patient was started on ceftriaxone, metronidazole, and gentamicin $160 \mathrm{mg}$, as well as antiseizure prophylaxis. He was only administered gentamicin for 5 days. Afterward, he was continued on ceftriaxone, metronidazole, and cloxacillin intravenous meningeal doses. Radiography revealed that the patient's chest was clear; however, he had mild circumcised homogeneous infiltrate in his right lower lobe that could have been fibrosis related to his prior tuberculosis infection. The patient was discharged from our institution after 5 weeks on oral antibiotics, including ciprofloxacin, cloxacillin, and metronidazole. At his 1-month follow-up at our outpatient clinic, the patient made a complete neurological recovery apart from ongoing seizures A postoperative axial cut CT scan of the brain showed good evacuation of the left-sided occipital abscess (Fig. 4).

\section{Discussion}

\section{Background of SDE}

An intracranial SDE is defined as a widespread collection of pus located in the subdural space between the dura mater and arachnoid mater. ${ }^{2,9,10,23-26}$ SDEs, although less common than BAs, still constitute approximately $20 \%$ of all localized intracranial infections. ${ }^{25}$ As reported in the literature, about $40-80 \%$ of patients with SDEs have otorhinologic infections, most frequently of the paranasal sinuses, which leads to bacterial seeding of the subdural space. ${ }^{21}$ It is reported that up to $20 \%$ of SDEs occur following head trauma or cranial surgical procedures. ${ }^{13,27,28}$

The symptoms associated with SDE correlate with the effect of the inflammatory mass in the subdural space and the related inflammation of the brain and meninges. SDE typically presents with symptoms such as fever, headache, nausea, vomiting, focal neurological deficits, seizures, and meningeal irritation even when they are small in size. ${ }^{8,29}$ Other examination findings include meningismus, contralateral hemiplegia, cranial nerve palsies, and papilledema. ${ }^{9,10}$ As reflected in the literature, SDEs tend to have a higher incidence in males, with a male-to-female ratio of $3: 1{ }^{29}$ Several SDEs are polymicrobial in origin, but most commonly they result from infection with microorganisms such as streptococci, staphylococci, Haemophilus influenzae, Streptococcus pneumoniae, and other gram-negative bacilli. ${ }^{9,24,30,31}$

In patients with suspected SDE, diagnostic neuroimaging with CT or MRI is recommended to localize the infection within the intracranial cavity, as well as laboratory work-up..$^{9,21,32,33}$ Bacterial organisms are scarcely isolated prior to surgical treatment due to the encapsulated nature of SDE. ${ }^{9,25}$ However, spinal puncture is not advised for the evaluation and diagnosis of SDE, because it can be life threatening to patients with elevated ICP and empyemas, thereby leading to mass effect.8,17,26 The mainstay of SDE treatment consists of combined empirical antimicrobial therapy and surgical intervention. $2,10,22,25,29,32,33$ Patients are rarely managed solely with antibiotics. ${ }^{25,28,33}$ Currently, recommendations for the management of intracranial SDE support multimodal treatment options to offer better results, decrease the incidence of recurrence, and prevent long-term complications or disability. Surgical treatment consists of burr hole aspiration or craniotomy for drainage and debridement of the SDE. ${ }^{18,24,26}$ It is recommended that the collection of pus obtained upon evacuation undergo laboratory evaluation for both aerobic and anaerobic cultures. ${ }^{13,25,27,32}$ Antibiotic therapy should be adjusted to account for reported microorganisms from culture results. ${ }^{8,9,29,34}$ Last, longer periods of 6-8 weeks of antimicrobial therapy should be administered in conjunction with anticonvulsant therapy, as well as close follow-up and monitoring of 

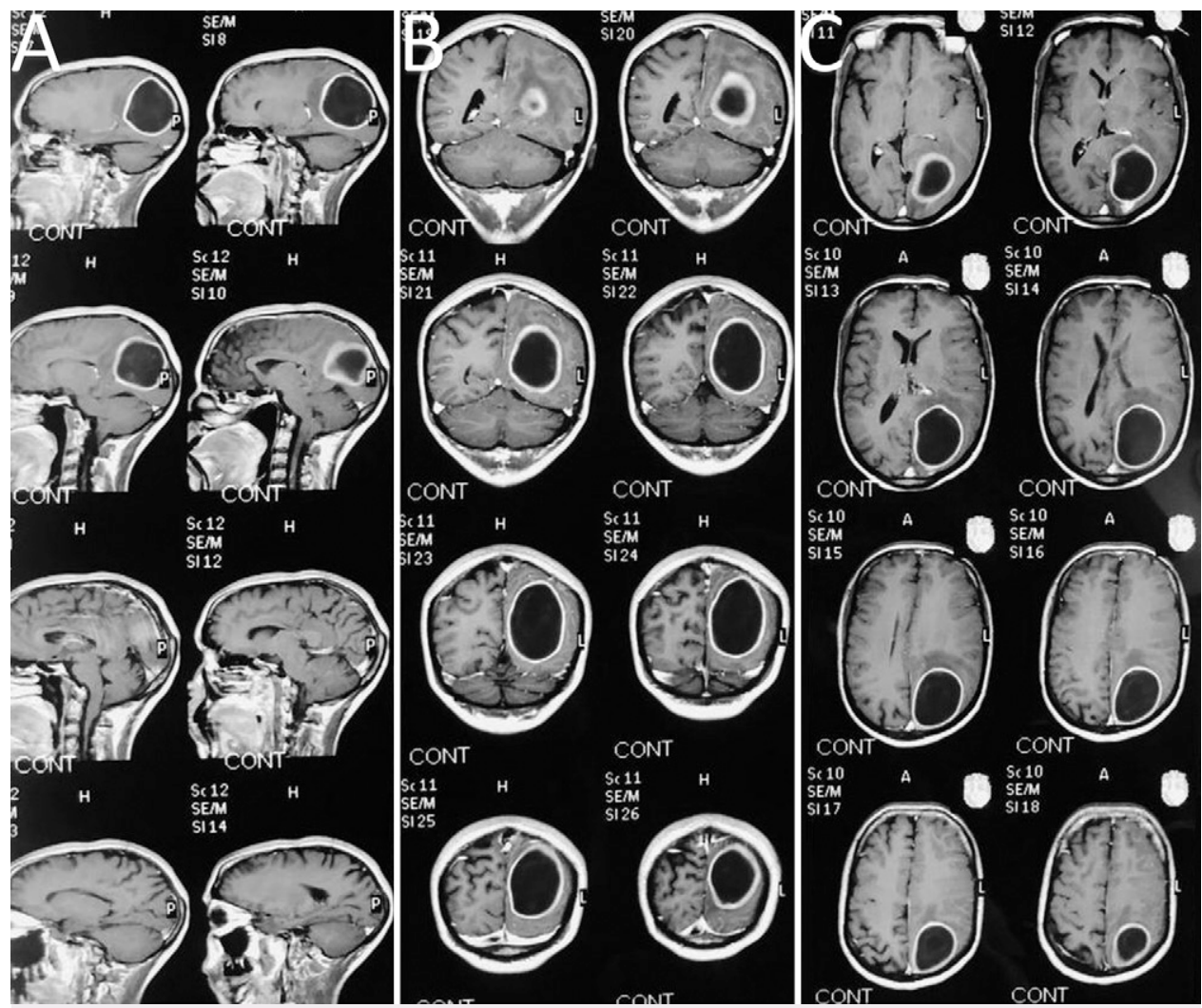

FIG. 3. Preoperative brain MRI scans show left-sided occipital brain abscess. A: Preoperative sagittal cut brain MRI scan. B: Preoperative coronal cut brain MRI scan. C: Preoperative axial cut brain MRI scan.
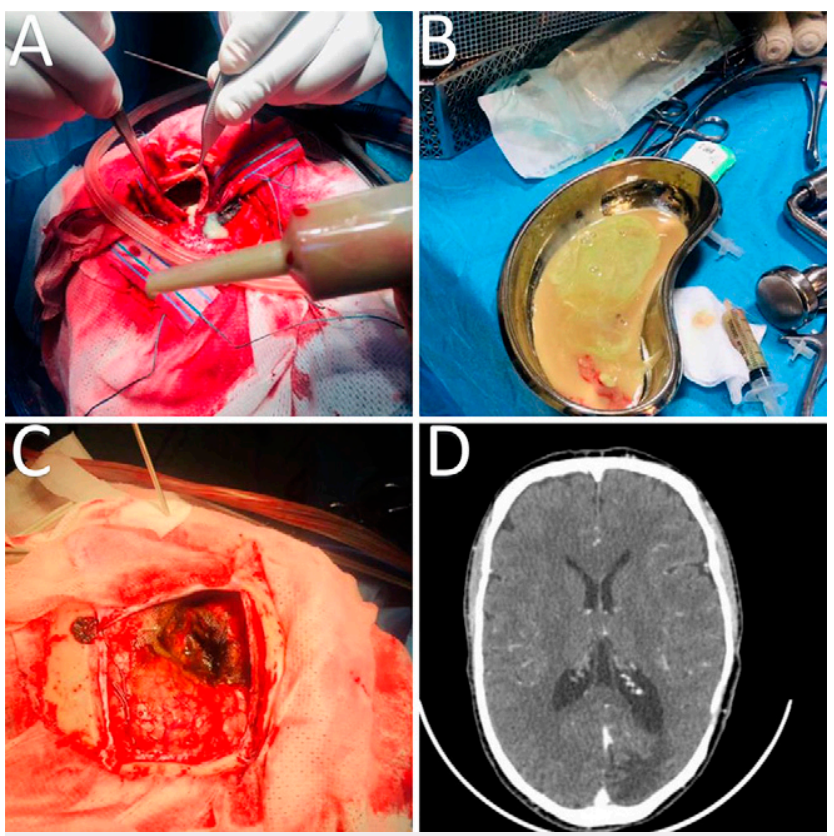

FIG. 4. A and B: Intraoperative drainage of the occipital brain abscess and evacuation of frank pus. C: Intraoperative finding after drainage of the intracranial suppuration revealed a thick capsule. D: Postoperative axial cut brain CT scan showed good evacuation of the abscess. laboratory values and neuroimaging to support favorable prognosis in patients with SDE. ${ }^{2,35-37}$

\section{Background of BA}

A BA is characterized as a localized area of necrosis within the brain parenchyma that results from a focal infection or traumatic process. $^{1,15}$ In low- and middle-income countries, BAs comprise a significant percentage of space-occupying intracranial lesions with an incidence of about $8 \%$, compared with an incidence of $1-2 \%$ in developed nations. ${ }^{38}$ Although the origin of BA development can be difficult to elucidate, it most commonly arises from chronic rather than acute infections of the middle ear. ${ }^{12,14,15,38-41}$ However, BA can also stem from infections in the head and neck region, including mastoiditis, paranasal sinus infection, infection from frontal or ethmoid sinuses, dental infection, or facial trauma. ${ }^{12,17,33,36,38,42}$ of note, various conditions can cause BA due to hematogenous seeding of the brain, including pulmonary infections, congenital heart disease, bacterial endocarditis, skin infections, pelvic infections, and intraabdominal infections. ${ }^{1,15,17,41}$ The most common microbial pathogens associated with BA are Staphylococcus and Streptococcus species, with Viridans streptococci and Staphylococcus aureus being the most frequent causes of BA. ${ }^{42}$ Prevalent symptoms include fever, headache, focal neurological deficit, pain localized to the side of the abscess, seizures, nausea, mental status changes, vomiting, and nuchal rigidity. ${ }^{1,2,14,17,20,39,40,42}$

Early detection and diagnosis of BA through CT or MRI allow better localization of the abscess. ${ }^{42}$ As with SDE, it is considered extremely 
dangerous to perform lumbar puncture to obtain cerebrospinal fluid cultures in patients with BA unless clinicians strongly suspect concurrent meningitis. ${ }^{1,15}$ Treatment for BA is similar to the treatment of SDE. After surgical drainage and evacuation of pus collected intraoperatively from BA, the pus should be cultured to obtain knowledge about the microbiology of $\mathrm{BA}$ and determine antibiotic sensitivity. ${ }^{3,14,39} \mathrm{~A}$ combination of a 6-week course of antimicrobial therapy with anticonvulsant therapy is recommended in patients. ${ }^{36,41,42}$ Last, all patients should be closely monitored with follow-up CT or MRI and postoperative laboratory evaluation until the BA has resolved. ${ }^{17,34,43}$

\section{Observations}

Through electronic database searching, a total of 98 papers were collected, and duplicate studies were removed from consideration. We identified 88 deduplicated works, of which 6 papers remained after title screening and abstract review. Ultimately, only 1 study, by Nour et al., met our inclusion criteria from our literature review (Supplementary Fig. 1). ${ }^{44}$

Nour et al. presented a case from their institution in which a male patient was diagnosed with chronic calcified SDE. ${ }^{44}$ This case report highlighted similar rare features seen in our patient cases of sterile ICS. The patient in the study by Nour et al. was observed to have nonspecific clinical signs and symptoms and no history of trauma, meningitis, tuberculosis, or previous surgery. ${ }^{44}$ Ultimately, no identifiable predisposing medical condition could be determined that could have been a potential cause for the development of the chronic calcified SDE. This patient had laboratory results that were within normal limits, and his social history was unrevealing. His vital signs, meningeal signs, and a chest radiograph were all unremarkable. The presentation of this patient reported by Nour et al. was comparable to our patients with ICS at our institution. ${ }^{44}$ Moreover, preoperative CT and MRI showed a left frontoparietal peripherally calcified, subdural hypodense collection eliciting mass effect on the adjacent brain parenchyma. ${ }^{44}$ The pus that was drained intraoperatively was evaluated for cell count, chemistry, and Gram stain culture; however, all results were unrevealing. ${ }^{44}$ Postoperatively, the patient's condition declined 2 days after surgical intervention, and he required emergency reoperation. ${ }^{44}$ Additional culture samples were obtained; however, both the original cultures and the reoperation cultures failed to reveal any microbial organism growth. ${ }^{44}$ The patient did receive high doses of broad-spectrum antibiotics throughout his course, but he did not recover and subsequently died 10 days after surgery. ${ }^{44}$

Although this patient reported by Nour et al. did not share similar clinical recovery outcomes to our case reports, it still showcases the rarity of sterile $\mathrm{ICS}^{44}$ It is uncommon that there are no detectable predisposing factors that lead to the development of the ICS, and it is even more unusual to see sterile site ICS. This literature review draws attention to the dearth of studies available regarding sterile ICS and corroborates the need for future studies to reliably compare case presentations and determine appropriate treatment for these uncommon cases.

\section{Lessons}

Uncommon Sterile Site of Cases/Similarities of Cases and Possible Causes

This report of 2 unconventional patient cases of ICS highlights 3 anomalies that are uncommonly seen in cases of ICS. Although ICS is considered a rare complication of infection, our 2 case reports share 3 similar features that make our study more unusual than most cases of ICS. First, both patients had no discernible history or conditions that are commonly considered to be predisposing factors for ICS development. Second, although some cases of ICS are associated with nonspecific sequelae such as abnormal vital signs or laboratory values such as fever, leukocytosis, serum CRP level, and erythrocyte sedimentation rate, our 2 cases did not present with these features. $2,5,7,11,16,17,19$ Last, both intraoperative cultures did not reveal microorganism growth and were deemed to be sterile. In case 2, the pus obtained from the BA capsule showed only 1 white blood cell present, and no causative pathogen could be identified. Although these 3 features are frequently seen separately in cases of ICS, it is rare for all 3 to be present concurrently.

A potential hypothesis to explain why our patients did not present with fever or elevated leukocyte counts is that it may be a result of localization of the ICS. Laboratory values of blood tests can be nonspecific and not pathognomonic for ICS. $9,14,25,32,33$ In addition, blood cultures have a poor yield as low as $5 \%$, which is also most likely due to the blood-brain barrier and enhanced localization of infection within the intracranial compartment, which is why intraoperative cultures should be obtained. ${ }^{9}$ Although no identifiable causes for ICS could be determined in our patient cases, other potential factors to consider include infection stemming from tattoo procedures in case 1 that spread to the intracranial compartment or related to history of tuberculosis infection in case 2 . Chen et al. reported a case of spinal SDE following acupuncture. ${ }^{45}$ This study may support our theory of needle contamination during tattoo procedures and suggests the importance of sterilization procedures to prevent ICS infection. $^{45}$

Another possible reason for the sterile site of ICS cases is that operative culture results can be negative without any reported microorganisms in up to $7-53 \%$ of patients..$^{9,10,35}$ This can be accounted for by the frequent use of preculture/preoperative antimicrobial therapy. ${ }^{9,35}$ It is not uncommon that responsible microorganisms for BA development are unable to be detected from the pus collection; however, the infection may still be present in the capsule of the abscess. ${ }^{9,15,38}$ This supports the potential for excision of the abscess capsule entirely instead of just aspiration to ensure improvement of a patient's condition and prevent persistent infection. ${ }^{17}$ Despite improved culturing techniques to detect the infectious pathogen, there are high rates of sterile cultures that have been reported by several other studies. ${ }^{40}$ It is speculated that because most cases are empirically treated with antibiotics, there is a high possibility for cultures to be negative. However, failure of culture growth may be a result of early intervention with empirical antibiotic treatment or improper culturing technique. ${ }^{9}$ Therefore, incorporation of molecular genetic tests to identify causative microorganisms should be a part of diagnosis. ${ }^{34}$

\section{Limitations}

The strengths and limitations of this study should be acknowledged. All operations were performed by one neurosurgeon with uniform operative techniques, thereby decreasing performance bias. However, there were only 2 patient cases presented in this singleinstitution evaluation, which limited the statistical power of this study. Last, the paucity of available literature regarding sterile ICS rendered our analyses limited. 


\section{Conclusions}

In summary, ICS is a rare but life-threatening infection that still presents as a public and global health concern. This report and literature review details 2 uncommonly encountered cases of sterile ICS in which both patients had no discernible preexisting conditions or history frequently seen with ICS. Sterile ICS deserves further investigation not only to elucidate its etiology but also to establish a standardized treatment paradigm to improve clinical outcomes.

\section{References}

1. Alvis Miranda $\mathrm{H}$, Castellar-Leones SM, Elzain MA, Moscote-Salazar LR. Brain abscess: current management. J Neurosci Rural Pract. 2013;4(1 suppl 1):S67-S81.

2. Tsai YD, Chang WN, Shen CC, et al. Intracranial suppuration: a clinical comparison of subdural empyemas and epidural abscesses. Surg Neurol. 2003;59(3):191-196.

3. Fiorindi A, Marton E, Gioffrè G, et al. New perspectives in the endoscopic treatment of brain purulent collections: targets, techniques, results in a case series, and overview of the literature. Acta Neurochir (Wien). 2020;162(11):2867-2874.

4. Loembe PM, Okome-Kouakou M, Alliez B. Suppurative intracranial infections in Africa. Article in French. Med Trop (Mars). 1997;57(2): 186-194.

5. Heineman HS, Braude Al, Osterholm JL. Intracranial suppurative disease. Early presumptive diagnosis and successful treatment without surgery. JAMA. 1971;218(10):1542-1547.

6. van der Velden FJS, Battersby A, Pareja-Cebrian L, Ross N, Ball SL, Emonts M. Paediatric focal intracranial suppurative infection: a UK single-centre retrospective cohort study. BMC Pediatr. 2019;19(1):130.

7. Yõldõrmak T, Gedik H, Simşek F, Kantürk A. Community-acquired intracranial suppurative infections: a 15-year report. Surg Neurol Int. 2014;5:142.

8. Bockova J, Rigamonti D. Intracranial empyema. Pediatr Infect Dis J. 2000;19(8):735-737.

9. Yoon J, O'Bryan C, Redmond M. Intracranial subdural empyema-a mini review. J Infect (Grand Rapids). 2020;3:1-5.

10. Dill SR, Cobbs CG, McDonald CK. Subdural empyema: analysis of 32 cases and review. Clin Infect Dis. 1995;20(2):372-386.

11. Djientcheu VP, Mouafo TF, Esiene A, et al. Intracranial suppurations in the African child: a severe but preventable complication. Childs Nerv Syst. 2013;29(1):119-123.

12. Helweg-Larsen J, Astradsson A, Richhall $H$, Erdal J, Laursen $A$, Brennum J. Pyogenic brain abscess, a 15 year survey. BMC Infect Dis. 2012;12(1):332.

13. Fernández-de Thomas RJ, De Jesus 0 . Subdural empyema. In: StatPearls. StatPearls Publishing; 2021.

14. Muzumdar D, Jhawar S, Goel A. Brain abscess: an overview. Int J Surg. 2011;9(2):136-144.

15. Mathisen GE, Johnson JP. Brain abscess. Clin Infect Dis. 1997; 25(4):763-781.

16. Leotta N, Chaseling R, Duncan G, Isaacs D. Intracranial suppuration. J Paediatr Child Health. 2005;41(9-10):508-512.

17. Oliver L. Intracranial suppuration. Postgrad Med J. 1961;37(431): 534-540.

18. Joubert MJ, Stephanov S. Computerized tomography and surgical treatment in intracranial suppuration. Report of 30 consecutive unselected cases of brain abscess and subdural empyema. J Neurosurg. 1977;47(1):73-78.

19. Anwary MA. Intracranial suppuration: review of an 8-year experience at Umtata General Hospital and Nelson Mandela Academic Hospital, Eastern Cape, South Africa. S Afr Med J. 2015;105(7): 584-588.
20. Adachi J, Uki J, Kazumoto K, Takeda F. Diagnosis of brainstem abscess in the cerebritis stage by magnetic resonance imagingcase report. Neurol Med Chir (Tokyo). 1995;35(7):467-470.

21. Weingarten K, Zimmerman RD, Becker RD, Heier LA, Haimes AB, Deck MD. Subdural and epidural empyemas: MR imaging. AJR Am J Roentgenol. 1989;152(3):615-621.

22. Nathoo N, Nadvi SS, van Dellen JR, Gouws E. Intracranial subdural empyemas in the era of computed tomography: a review of 699 cases. Neurosurgery. 1999;44(3):529-536.

23. Al Salihi M, Kheiri B, Abu Sitta E, Kanzy A. Sterile subdural empyema: an unusual presentation. BMJ Case Rep. 2017;2017:bcr2017222053.

24. Roos KL. Subdural empyema. In: Aminoff MJ, Daroff RB, eds. Encyclopedia of the Neurological Sciences. 2nd ed. Academic Press; 2014:335-336.

25. French H, Schaefer N, Keijzers G, Barison D, Olson S. Intracranial subdural empyema: a 10-year case series. Ochsner J. 2014;14(2): 188-194.

26. Chikani MC, Mezue W, Okorie E, Mbachu C, Ndubisi C, Chikani UN. Subdural empyema: clinical presentations and management options for an uncommon neurosurgical emergency in a developing country. Niger J Clin Pract. 2017;20(10):1221-1225.

27. Silverberg AL, DiNubile MJ. Subdural empyema and cranial epidural abscess. Med Clin North Am. 1985;69(2):361-374.

28. Wu TJ, Chiu NC, Huang FY. Subdural empyema in children-20year experience in a medical center. $J$ Microbiol Immunol Infect. 2008:41(1):62-67.

29. De Bonis P, Anile C, Pompucci A, Labonia M, Lucantoni C, Mangiola A. Cranial and spinal subdural empyema. Br J Neurosurg. 2009;23(3) 335-340.

30. Yoshikawa TT, Chow AW, Guze LB. Role of anaerobic bacteria in subdural empyema. Report of four cases and review of 327 cases from the English literature. Am J Med. 1975;58(1):99-104.

31. Merad S. Anaerobic bacteria in intracranial suppuration. Article in French. Arch Inst Pasteur Alger. 1989;57:267-276.

32. Weisberg L. Subdural empyema. Clinical and computed tomographic correlations. Arch Neurol. 1986;43(5):497-500.

33. Widdrington JD, Bond $H$, Schwab U, et al. Pyogenic brain abscess and subdural empyema: presentation, management, and factors predicting outcome. Infection. 2018;46(6):785-792.

34. Udayakumaran S, Joseph T. A proposal for a tailored protocol for focal suppurative infection of the central nervous system: analysis of an institutional experience in pediatric patients. Neurosurg Focus. 2019;47(2):E11.

35. Mauser HW, Tulleken CA. Subdural empyema. A review of 48 patients. Clin Neurol Neurosurg. 1984;86(4):255-263.

36. Bernardini GL. Diagnosis and management of brain abscess and subdural empyema. Curr Neurol Neurosci Rep. 2004;4(6):448-456.

37. Greenlee JE. Subdural empyema. Curr Treat Options Neurol. 2003;5(1):13-22.

38. Moorthy RK, Rajshekhar V. Management of brain abscess: an overview. Neurosurg Focus. 2008;24(6):E3.

39. Sharma BS, Khosla VK, Kak VK, et al. Multiple pyogenic brain abscesses. Acta Neurochir (Wien). 1995;133(1-2):36-43.

40. Tfifha M, Abdallah AB, Saadaoui K, et al. Pyogenic brain abscess in children: a Tunisian multi-centric experience. Arch Pediatr Infect Dis. 2018;6(3):e55012.

41. Hakan T. Management of bacterial brain abscesses. Neurosurg Focus. 2008;24(6):E4.

42. Chowdhury FH, Haque MR, Sarkar MH, Chowdhury SMNK, Hossain Z, Ranjan S. Brain abscess: surgical experiences of 162 cases. Neuroimmunol Neuroinflamm. 2015;2:153-161.

43. Boukassa L, Ngackosso O, Kinata S, Ekouele Mbaki H. Intracranial suppurations in Africa: systematic review. Open J Mod Neurosurg. 2020;10:244-253. 
44. Nour AS, Shumbash KZ. Giant chronic calcified subdural empyema: a case report. J Med Case Rep. 2020;14(1):95.

45. Chen $\mathrm{MH}$, Chen $\mathrm{MH}$, Huang JS. Cervical subdural empyema following acupuncture. J Clin Neurosci. 2004;11(8):909-911.

\section{Disclosures}

The authors report no conflict of interest concerning the materials or methods used in this study or the findings specified in this paper.

\section{Author Contributions}

Conception and design: Karekezi, Pradhan, Munyemana. Acquisition of data: all authors. Analysis and interpretation of data: Karekezi,

Pradhan, Munyemana. Drafting the article: Pradhan, Munyemana.

Critically revising the article: all authors. Reviewed submitted version of manuscript: all authors. Approved the final version of the manuscript on behalf of all authors: Karekezi. Statistical analysis: Munyemana. Administrative/technical/material support: Munyemana. Study supervision: Karekezi, Rutayisire, Munyemana.

\section{Supplemental Information}

Online-Only Content

Supplemental material is available with the online version of the article. Supplementary Fig. 1. https://thejns.org/doi/suppl/10.3171/ CASE21570.

\section{Correspondence}

Correspondence: Claire Karekezi: Rwanda Military Hospital, Kicukiro District, Kigali City, Rwanda. clairekarekezi@gmail.com. 